\title{
Metabolic engineering of Escherichia coli for de novo production of betaxanthins
}

Yanan Hou ${ }^{1}$, Xue Liu ${ }^{1}$, Shilin $\mathrm{Li}^{1}$, Xue Zhang ${ }^{1}$, Sili Yu ${ }^{1}$, Guang-Rong Zhao*1,2

${ }^{1}$ Frontier Science Center for Synthetic Biology and Key Laboratory of Systems Bioengineering (Ministry of Education), School of Chemical Engineering and Technology, Tianjin University, Yaguan Road 135, Jinnan District, Tianjin 300350, China

${ }^{2}$ SynBio Research Platform, Collaborative Innovation Centre of Chemical Science and Engineering (Tianjin), Tianjin University, Yaguan Road 135, Jinnan District, Tianjin 300350, China

*Corresponding author: grzhao@tju.edu.cn

Tel: +86-22-85356580; Fax: +86-22-27403389 
Table S1. Primers used in this study.

\begin{tabular}{|c|c|}
\hline Primers & Sequence \\
\hline MjDO-Nc-F & CATGCCATGGGCATGAAAGGGAC \\
\hline MjDO-Ba-R & CGCGGATCCTTAGTCCGTTTTTTGAGTA \\
\hline BvDOBm-F & GGGAATTCCATATGAAACCAGAAGATTTCCG \\
\hline BVDOHd-R & CGGGGTACCTTATTAAATCGCAGCTTCCATTT \\
\hline YDBm-F & CGCGGATCCGATGTCTTCAACACGTATGCC \\
\hline YDHd-R & CCCAAGCTTTTATTAGCCTATCTGCACCGACA \\
\hline HpaNd-F & GGGAATTCCATATGAAACCAGAAGATTTCCG \\
\hline HpaKp-R & CGGGGTACCTTATTAAATCGCAGCTTCCATTT \\
\hline hisD-F & TCGCCATGAGCTTTAACACAAT \\
\hline armhisD-R & TACAGCCCGCAATACTCGCC \\
\hline armhisL-F & GTAATAAGGATCGGGGCATG \\
\hline hisG-R & TCACТССАТСАТСТТСТСААТСG \\
\hline hisG271-F & TGGTCAGCAGCAAAACCCTGTTCTGG \\
\hline hisG271-R & CCAGAACAGGGTTTTGCTGCTGACCA \\
\hline FRT(hisG)-F & CGATTGAGAAGATGATGGAGTGATGTGTAGGCTGGAGCTGCTTCG \\
\hline FRT(hisD)-R & ATTGTGTTAAAGCTCATGGCGAAATATCCTCCTTAGTTCCTATTCC \\
\hline \multirow{2}{*}{$\operatorname{arm}($ hisL')-R } & TGTGAAGCCTGCTTTTTTATACTAACTTGAGCGTTAAATTGAACGCGTGTCA \\
\hline & $\mathrm{T}$ \\
\hline
\end{tabular}




\begin{tabular}{ll}
\hline & GTTAGTATAAAAAAGCAGGCTTCACAGGAGACTTTCTGATGACAGACAAC \\
hisG(hisL')-F & ACTCGTTTACG \\
hisG(hind)-R & CATTATGCGGCCGCAAGCTTTCACTCCATCATCTTCTCAATCG \\
hisL'(pst)-F & CGAGCTCGGCGCGCCTGCAGATGACACGCGTTCAATTTAAC \\
DODtest-F & GATCCAGTATCGCGCACCG \\
DODtest-R & CGAATACGCCGTTGGCCGCC \\
HindIII-F & AAGCTTGCGGCCGCATAATG \\
PstI-R & CTGCAGGCGCGCCGAGCTCG \\
\hline
\end{tabular}

Table S2. Codon-optimized nucleotide sequences of genes $B v D O D A$ and $M J D O D A$ in this study.

\section{Optimized Sequences}

\section{Gene}

BvDoda ATGGGCAGCGAAGACAACATCAAAGAAACTTTCTTCATCTCGCACGGCAC

TCCGATGATGGCCATTGATGACTCCAAACCATCTAAAAAATTTTTAGAGTCT

TGGCGTGAAAAAATCTTTTCTAAGAAACCGAAAGCCATTTTAGTCATCTCC

GCACACTGGGAAACCGATCAGCCGTCTGTGAACGTAGTAGACATTAACGA

CACTATCTACGACTTCCGAGGTTTTCCGGCGCGTCTGTACCAGTTCAAATAC

TCTGCGCCGGGCTCACCTGAACTGGCGAACCGTATTCAGGATCTGCTGGCC

GGTTCTGGCTTCAAAAGCGTCAATACCGATAAAAAACGTGGCTTGGATCAC

GGCGCGTGGGTTCCCCTGATGCTGATGTATCCAGAGGCGGATATTCCGGTTT 
GCCAATTATCGGTCCAGAGCCATTTAGACGGTACACACCATTACAAACTGG

GTCAGGCGCTGGCGCCGCTGAAGGATGAAGGTGTCCTGATCATCGGTAGC

GGCAGCGCAACCCACCCGTCCAACGGCACTCCGCCGTGCTCCGATGGTGT

CGCACCTTGGGCTGCTGCCTTTGATTCATGGTTAGAGACCGCTCTGACTAA

CGGCTCCTATGAAGAAGTCAATAAATATGAGACCAAGGCCCCAAACTGGA

AACTGGCTCACCCTTGGCCGGAACACTTCTACCCGCTCCACGTTGCGATGG

GTGCGGCAGGCGAAAACAGCAAAGCCGAACTGATCCACAACTCGTGGGA

CGGTGGTATTATGTCCTACGGTTCTTATAAATTCACATCCACCTAA

MjDoda

ATGAAAGGGACATACTATATTAATCACGGCGACCCTCTGATGTACCTGAAAA

AGCACATTAAATTGCGTCAGTTCCTGGAGGGTTGGCAGGAGAACGTTGTG

ATCGAAAAGCCGAAGTCCATTCTGATCATTTCCGCCCATTGGGACACTAAC

GTGCCGACTGTGAACTTCGTGGAACATTGCGACACCATCCACGATTTCGAC

GACTACCCGGACCCACTGTATCAGATCCAGTATCGCGCACCGGGTGCTCCT

AACCTGGCTAAAAAAGTTGAGGAGCTGCTGAAAGAGTCTGGTATGGAGTG

CGAAATTGATACCAAACGTGGTCTGGATCACGCAGCGTGGTTCCCGCTGAT

GTTCATGTATCCGGAAGCTAATATTCCTATTTGTGAACTCTCTGTTCAGCCG

AGCAAAGACGGTATTCACCATTACAACGTGGGCAAGGCACTGTCACCGCT

GCTCCAACAGGGTGTTCTGATCATCGGTTCGGGCGGCACCGTTCACCCGAG

CGACGACACCCCGCACTGCCCGAACGGTGTTGCGCCCTGGGCTATCGAGT

TTGATAACTGGCTGGAAGATGCCCTGCTGTCTGGTCGTTACGAAGATGTTA 
ACAACTTTAAAAAGCTGGCACCGAACTGGGAAATCTCCCACCCGGGCCAG

GAGCACCTGTATCCGCTGCACGTTGCACTGGGCGCGGCTGGTAAAAACCC

GAAAACCCAACTGATTCATCGTTCCTGGGCGGCCAACGGCGTATTCGGTTA

TAGCACTTACAACTTCACCCCTACTACTCAAAAAACGGACTAA

Table S3. Sequence of his $L$ along with the downstream noncoding region before $h i s G$.

\begin{tabular}{|c|c|}
\hline Gene & Sequence \\
\hline hisL along with the & ATGACACGCGTTCAATTTAAACACCACCATCATCACCATCATCCT \\
\hline downstream noncoding & GACTAGTCTTTCAGGCGATGTGTGCTGGAAGACATTCAGATCTT \\
\hline \multirow[t]{3}{*}{ region before his $G$} & CCAGTGGTGCATGAACGCATGAGAAAGCCCCCGGAAGATCACC \\
\hline & TTCCGGGGGCTTTTTTATTGCGCGGTTGATAACGGTTCAGACAG \\
\hline & GTTTAAAGAGGAATAACAAA \\
\hline
\end{tabular}

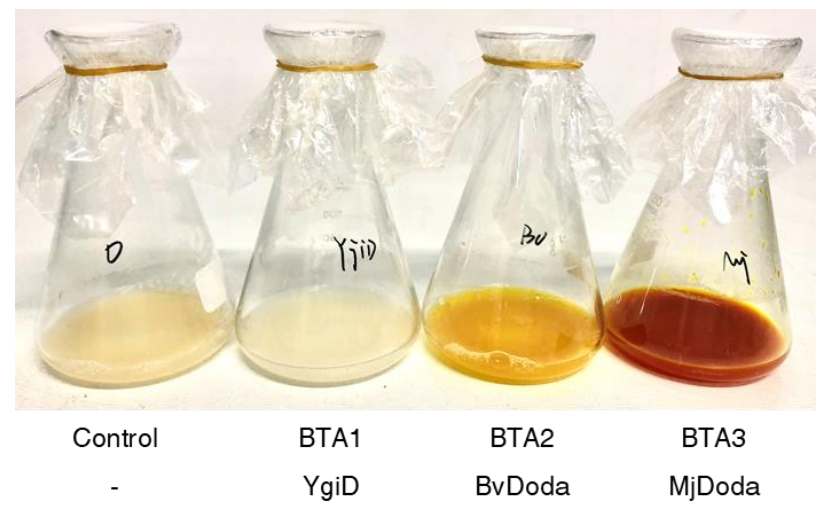

Figure S1. Visible color differences among strains BTA1, BTA2 and BTA3. 

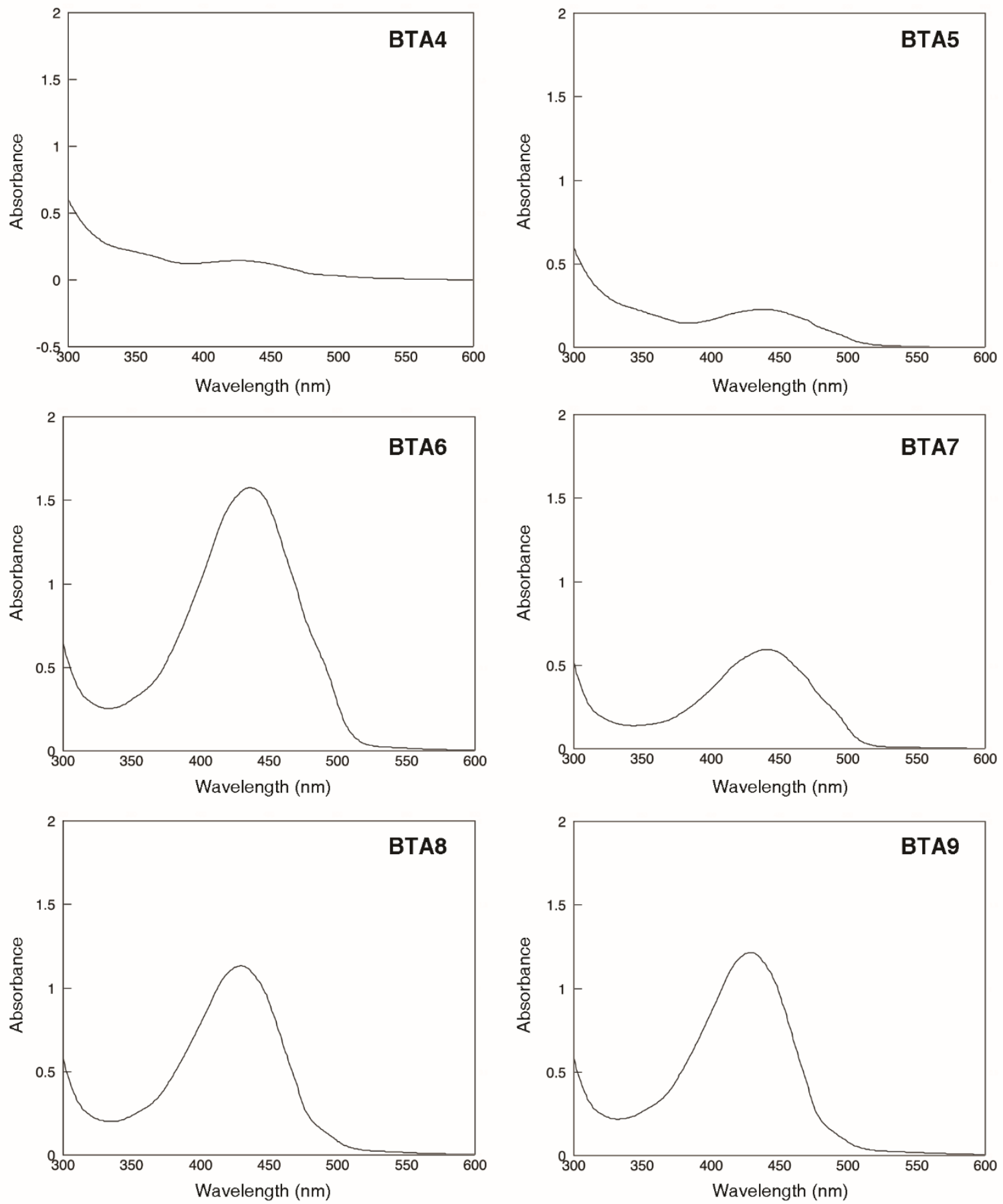

Figure S2. UV-visible spectra of the supernatants of strains BTA4-BTA9. 
(1)

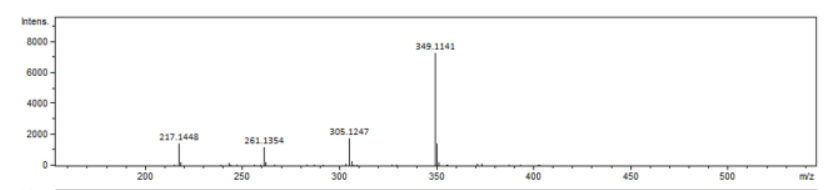

(2a)

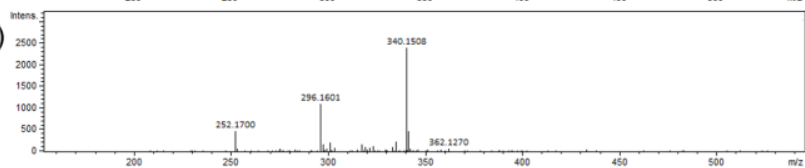

(2b)

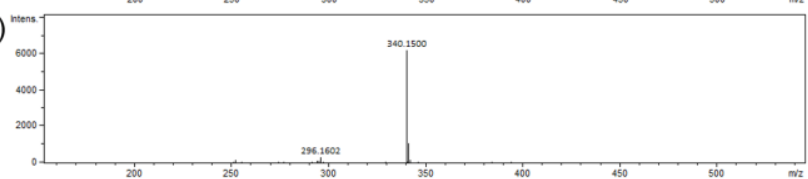

(3)

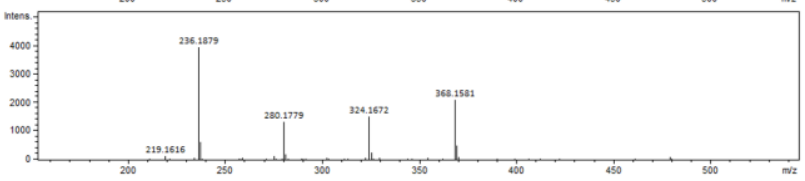

(4)

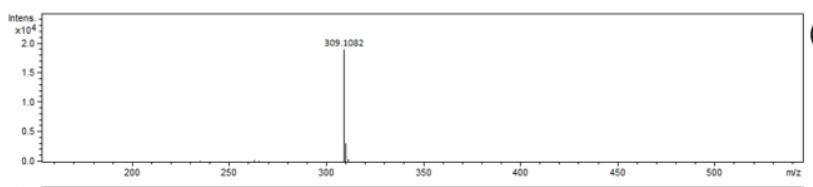

(5)

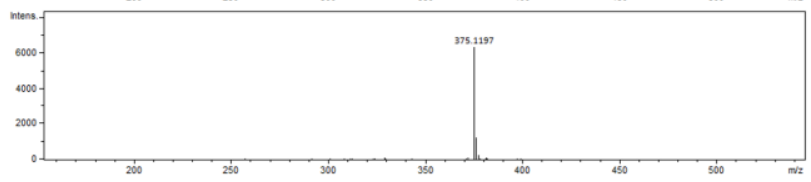

(6)

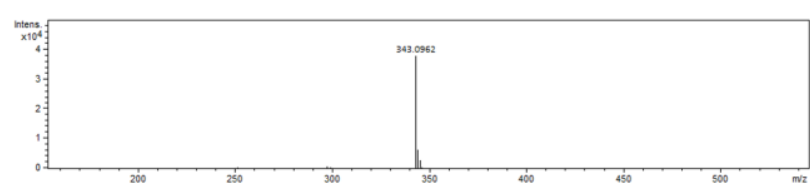

(7)

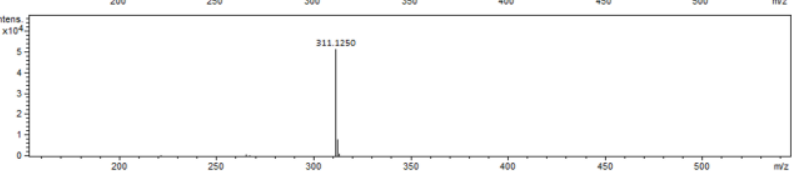

(8)

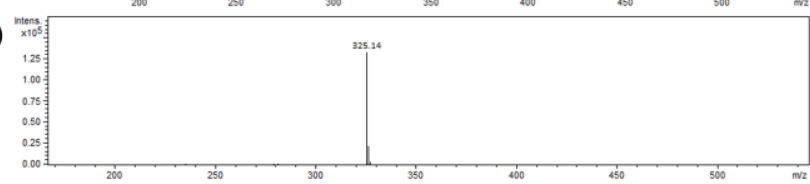

(9)

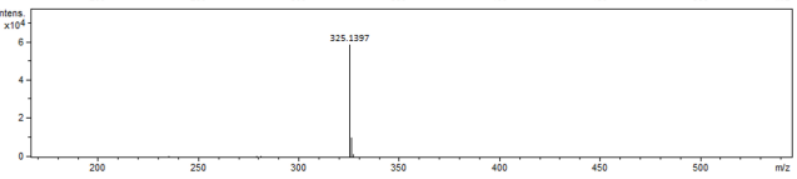

(10) $)^{\mathrm{m} x}$

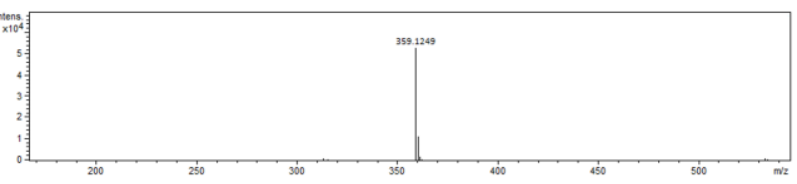

Figure S3. Mass spectra of different amino acid-betaxanthins.

From 1 to 10: Histidine-betaxanthin, Lysine-betaxanthin, Arginine-betaxanthin, Proline-betaxanthin, Tyrosine-betaxanthin, Methionine-betaxanthin, Valine-betaxanthin, Isoleucine-betaxanthin, Leucinebetaxanthin, Phenylalanine-betaxanthin ( $2 \mathrm{a}$ and $2 \mathrm{~b}$ corresponds to the two peaks at $4.73 \mathrm{~min}$ and $7.25 \mathrm{~min}$ in HPLC analysis) 


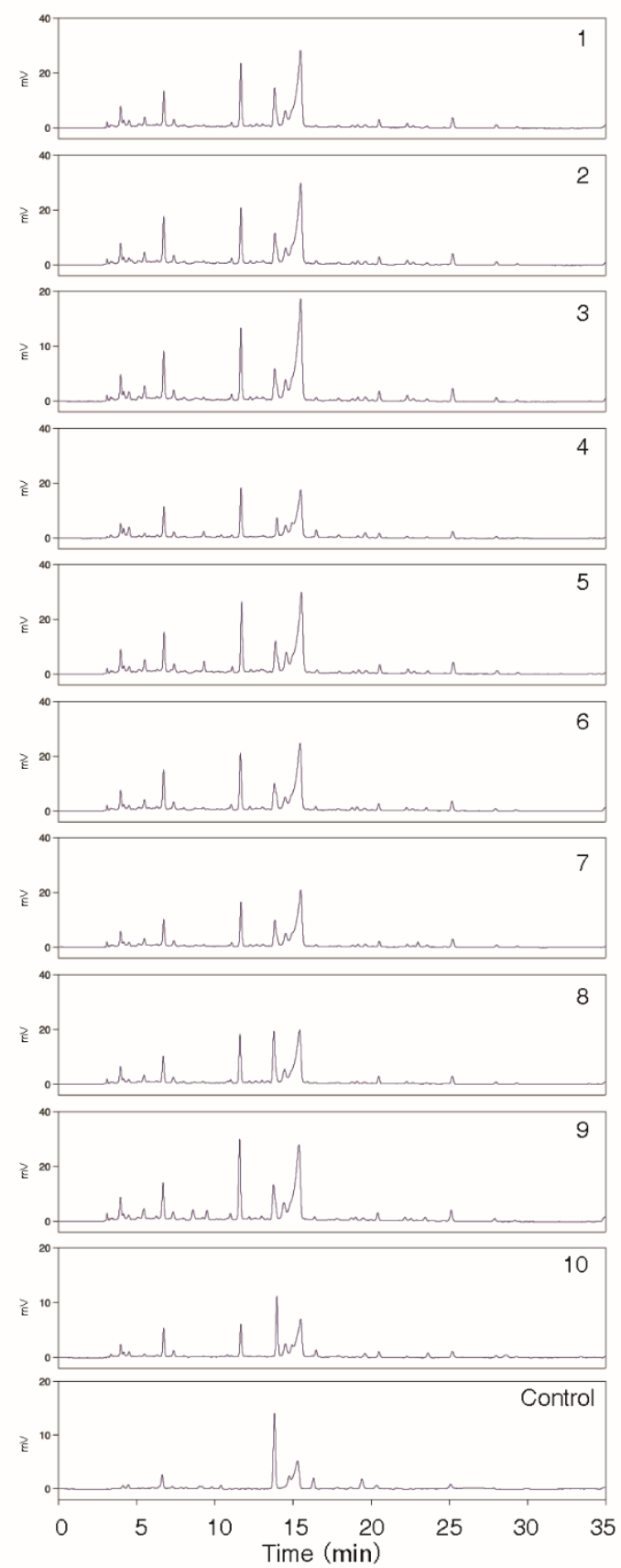

Figure S4. HPLC spectra of the supernatants of feeding of 10 protein amino acids failed to form betaxanthins.

From 1 to 10: L-alanine, L-asparagine, L-aspartic acid, L-cysteine, L-glutamic acid, L-glutamine, Lglycine, L-serine, L-threonine, L-tryptophan 


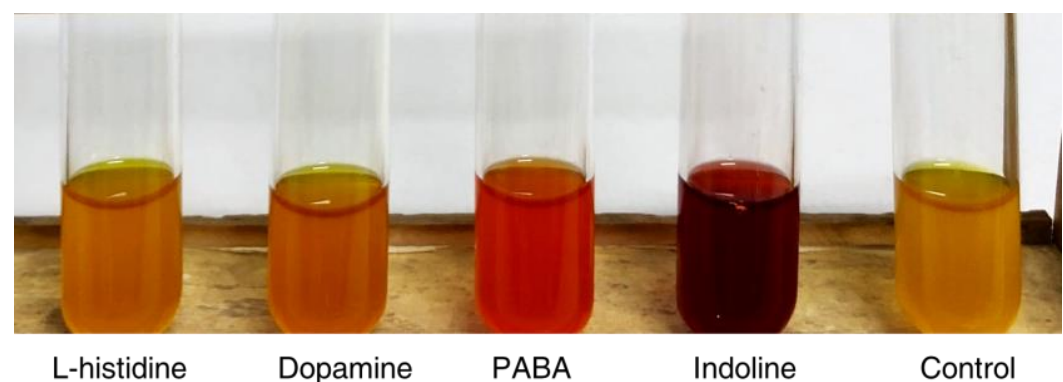

Figure S5. Examples of visible color differences of the feeding assays.

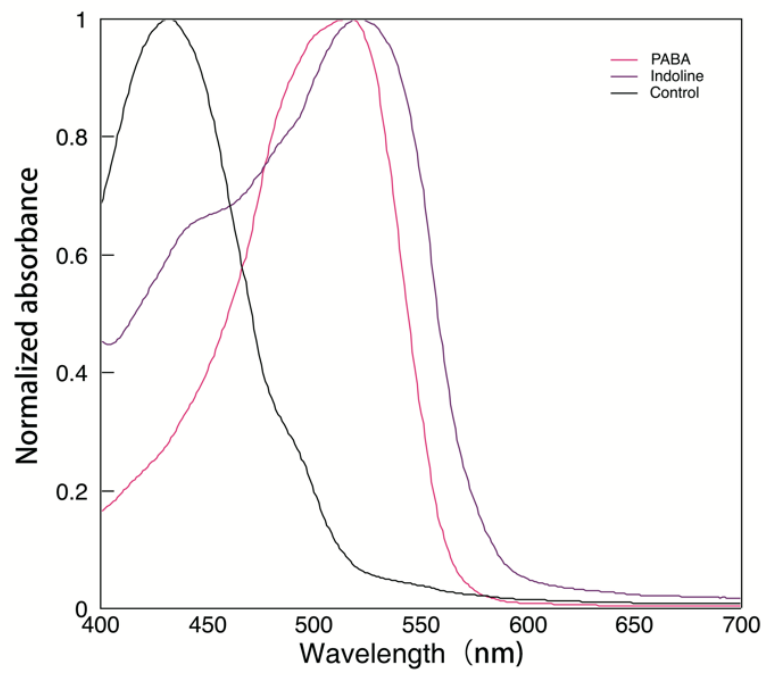

Figure S6. Normalized absorbance curves of supernatants feeding with PABA and indoline by UVvisible spectrophotometer analysis. 
(1)

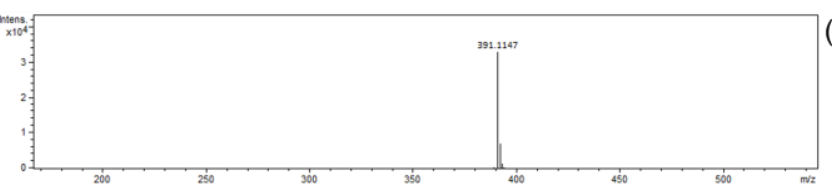

(2)

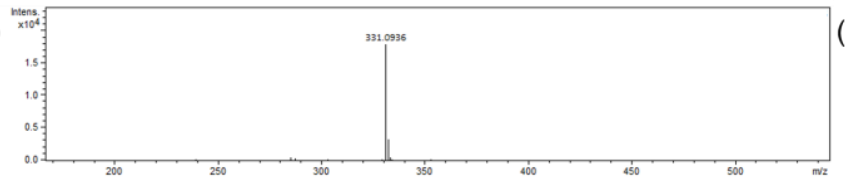

(3)

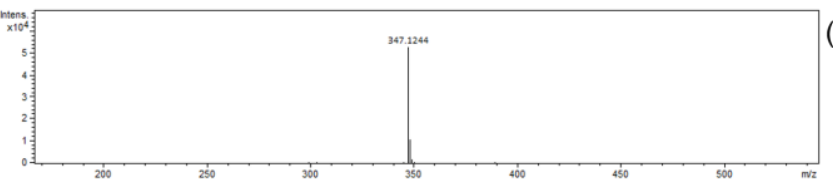

(4)

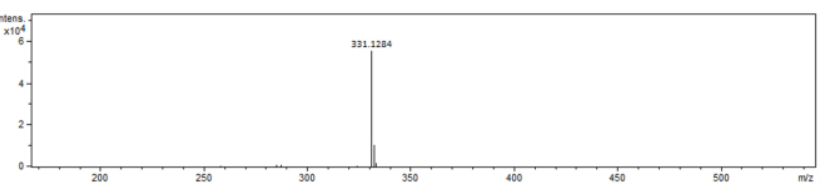

(5)
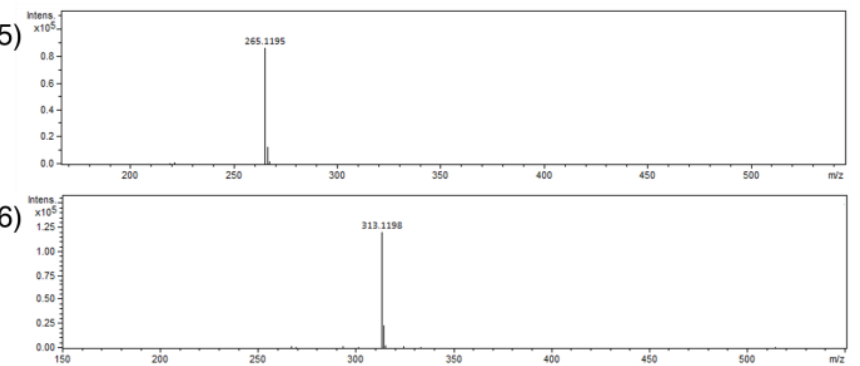

Figure S7. Mass spectra of different amino acid- and amine-betaxanthins.

From 1 to 6: Dopa-betaxanthin, PABA-betaxanthin, Dopamine-betaxanthin, Tyramine-betaxanthin, Pyrrolidine-betaxanthin, Indoline-betaxanthin 